\title{
Projeto de um controlador estabilizante usando parametrização de Youla aplicado em uma planta industrial
}

\author{
Renan L. Pereira* Karl H. Kienitz* Anderson T. de Moraes* \\ * Instituto Tecnológico de Aeronáutica, Praça Marechal Eduardo \\ Gomes, Vila das Acácias, 12228-900 São José dos Campos, SP, Brazil, \\ E-mails: \{renanrlp,kienitz,andtiago\}@ita.br
}

\begin{abstract}
This paper presents a practical investigation of a stabilizing controller via Youla parameterisation in an industrial level plant. The proposed control strategy consists of the combination of the concepts of doubly coprime factorization and Youla parameterisation. Differently from other reports in the literature, the synthesis of doubly coprime factorization is done by minimizing the $\mathcal{H}_{2}$-norm while the Youla parameter is determined using the $\mathcal{H}_{\infty}$ norm. Synthesis conditions are derived using LMIs to ensure the stability and performance of the closed-loop system. The experimental results showed that the proposed procedure can be an advantageous alternative for the synthesis of stabilizing controllers.

Resumo: Este artigo apresenta uma investigação prática de um controlador estabilizante via parametrização de Youla em uma planta industrial de nível. A estratégia de controle proposta consiste na combinação dos conceitos de fatoração duplamente coprima e parametrização de Youla. Diferentemente de outros trabalhos na literatura, a síntese da fatoração duplamente coprima é feita a partir da minimização da norma $\mathcal{H}_{2}$ enquanto que o parâmetro de Youla é determinado usando a norma $\mathcal{H}_{\infty}$. As condições de síntese são derivadas usando LMIs de forma a garantir a estabilidade e desempenho do sistema em malha fechada. Os resultados experimentais mostraram que o procedimento proposto pode ser uma alternativa interessante para a síntese de controladores estabilizantes.
\end{abstract}

Keywords: Doubly coprime factorization, Youla parameterisation, industrial plant, LMIs .

Palavras-chaves: Fatoração duplamente coprima, parametrização de Youla, planta industrial, LMIs.

\section{INTRODUÇÃO}

Descrições de sistemas usando fatoração coprima têm desempenhado um papel importante para o desenvolvimento da teoria de controle moderna. Muito desse interesse se refere ao estudo de alguns problemas fundamentais, como o problema de redução de modelos (Meyer, 1990; McFarlane et al., 1990), detecção de falha (Marx et al., 2003) e síntese de controladores robustos para sistemas lineares (Glover and McFarlane, 1989; McFarlane and Glover, 1992; Prempain and Postlethwaite, 2005) e não lineares (Verma and Hunt, 1993; Chen and Han, 1998).

Tendo em vista a síntese de controladores lineares, uma abordagem bastante utilizada é a parametrização de Youla (Youla et al., 1976). Ela permite através de uma fatoração duplamente coprima determinar um conjunto de controladores dinâmicos de saída estabilizantes (Zhou et al., 1996). Nesta abordagem os sistemas em malha fechada realizáveis são afins e descritos por um parâmetro $Q$ dinâmico livre estável. Uma estrutura de projeto de sistema de controle baseada nessa descrição é chamada de "abordagem de parâmetros Q". Vários projetos de sistemas de controle resolvem o problema de satisfazer os requisitos de desem- penho ao especificar esse parâmetro $Q$. Dentre estes projetos, nós podemos destacar as estratégias desenvolvidas por Moore et al. (1990) onde uma classe de controladores estabilizantes pode ser estruturada através das estimativas de estado, Kabamba et al. (1994) onde é apresentada a síntese de controladores vibracionais estabilizantes para sistemas SISO e Niemann (2003) que usando a parametrização dual de Youla apresenta um aspecto diferente de usar a parametrização de todos os sistemas estabilizados por um determinado controlador.

Como se pode observar, não existe uma estratégia única que permita satisfazer os requisitos de desempenho e estabilidade usando a parametrização de Youla. Neste sentido, a contribuição deste artigo consiste na investigação prática de um controlador estabilizante via parametrização de Youla em uma planta industrial de nível. A estratégia de controle proposta consiste na combinação dos conceitos de fatoração duplamente coprima e parametrização de Youla. Aqui, a síntese da fatoração duplamente coprima é feita a partir da minimização da norma $\mathcal{H}_{2}$ enquanto que o parâmetro de Youla é determinado usando a norma $\mathcal{H}_{\infty}$. Ambas as condições de síntese são derivadas usando LMIs de forma a garantir a estabilidade e desempenho do sistema em malha fechada. A eficácia do método é avaliada a partir da implementação prática em uma planta 
industrial de nível produzida pela empresa Yokogawa ${ }^{\circledR}$ que tem sido amplamente empregada em outros artigos científicos (Pereira et al., 2016; Moraes and Kienitz, 2017).

Este trabalho está organizado da seguinte forma. Na seção 2, apresenta-se a descrição da planta piloto industrial. $\mathrm{Na}$ seção 3, resultados preliminares para determinar um controlador estabilizante via parametrização de Youla é fornecido. $\mathrm{Na}$ seção 4, os principais resultados para a síntese de um controlador estabilizante combinando a minimização das normas $\mathcal{H}_{2}$ e $\mathcal{H}_{\infty}$ são apresentados $\mathrm{Na}$ seção 5 , descreve-se as etapas de projeto da estratégia proposta e consequentemente os resultados experimentais obtidos. Na seção 6, apresenta-se a conclusão.

A notação usada aqui será: $\mathbb{R}^{n \times m}$ denota o conjunto de matrizes reais $n \times m, I_{n}$ é uma matriz identidade $n \times$ n. $M>0$ (ou $M<0)$ significa que $M$ é simétrica e positiva (ou negativa) definida. A notação $G:=\left[\begin{array}{c|c}A & B \\ \hline C & D\end{array}\right]$ é utilizada para representar uma realização em função transferência de $G$, dada por $G:=C(s I-A)^{-1} B+D$. Além disso, o espaço $R \mathcal{H}_{\infty}$ denota o espaço de sistemas sem polos no semiplano direito.

\section{DESCRIÇÃO DA PLANTA INDUSTRIAL}

A planta industrial de nível, que é o objeto do estudo de caso neste trabalho, foi fabricada pela Yokogawa ${ }^{\circledR}$ para testar algoritmos industriais de monitoramento e controle de pequena escala. A planta possui 3 tanques, chamados tanque de reserva (TQR), tanque 1 (TQ1) e tanque 2 (TQ2). Através dos tubos que conectam TQR a TQ1 e TQ2, é possível transferir o fluido entre eles com o auxílio de bombas acionadas por dois motores elétricos (Figuras 1 e 2). Além disso, o sistema a ser controlado possui duas

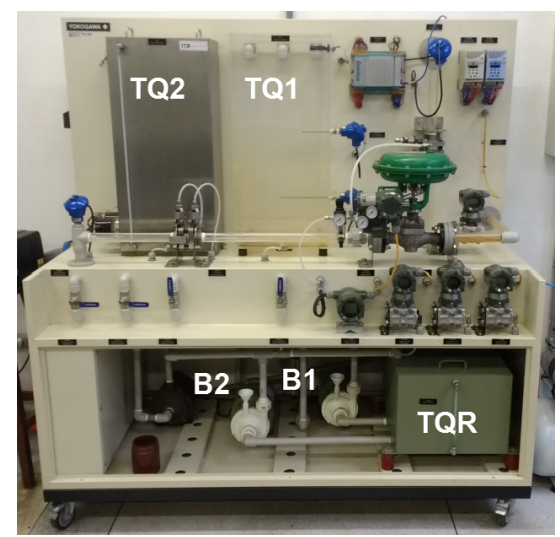

Figura 1. Planta industrial.

entradas (B1, B2) que representam os pontos de ajuste da bomba e duas saídas (H1 e H2) que correspondem ao nível no tanque 1 e no tanque 2, respectivamente. $\mathrm{O}$ modelo linear da planta industrial em torno dos pontos de operação relevantes pode ser obtido usando a teoria de modelagem de sistemas. Neste estudo de caso, a estrutura do modelo de Moraes and Kienitz (2017) foi escolhida. O modelo linear escolhido em espaço de estados é dado por

$$
\begin{aligned}
& \dot{x}(t)=A x(t)+B_{u} u(t) \\
& y(t)=C_{y} x(t)
\end{aligned}
$$

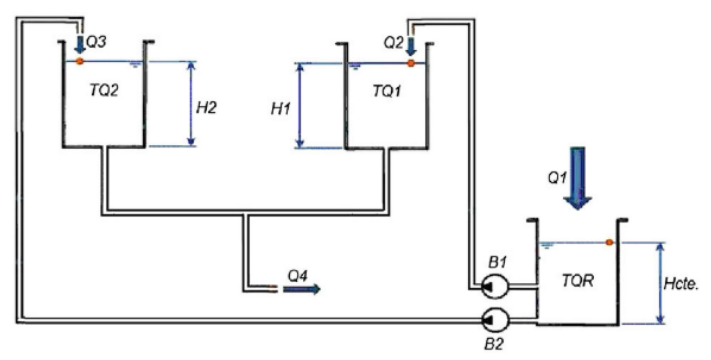

Figura 2. Diagrama do processo a ser controlado.

com

$$
A=\left[\begin{array}{cccc}
0 & -8,784.10^{-8} & 0 & 0 \\
1,318.10^{5} & -5,805 & 0 & -8,034 \\
0 & 0 & 0 & -1,043.10^{-7} \\
0 & -5,141 & 1,318.10^{5} & -11,18
\end{array}\right]
$$

$B_{u}=\left[\begin{array}{cc}1,58.10^{-7} & 0 \\ 0 & 0 \\ 0 & 2,45.10^{-7} \\ 0 & 0\end{array}\right] C_{y}=\left[\begin{array}{cccc}1,32.10^{5} & 0 & 0 & 0 \\ 0 & 0 & 1,32.10^{5} & 0\end{array}\right]$

Este modelo foi formulado com a ajuda de uma transformação de domínio (hidráulico para elétrico), e seu vetor de estado é definido como

$$
x^{T}(t)=\left[\begin{array}{llll}
q_{2} & \lambda_{5} & q_{7} & \lambda_{11}
\end{array}\right]
$$

sendo que $q_{2}$ e $q_{7}$ representam as cargas, enquanto $\lambda_{5}$ e $\lambda_{11}$ são os fluxos. Maiores detalhes sobre a modelagem do sistema pode ser encontrado em (Moraes and Kienitz, 2017).

\section{PRELIMINARES E DEFINIÇÃO DO PROBLEMA}

Considere o seguinte sistema linear invariante no tempo,

$$
\left[\begin{array}{l}
\dot{x} \\
z \\
y
\end{array}\right]=P\left[\frac{x}{w}\right]
$$

sendo $P$ uma planta generalizada dada por

$$
P=\left[\begin{array}{c|cc}
A & B_{w} & B_{u} \\
\hline C_{z} & D_{w z} & D_{u z} \\
C_{y} & D_{w y} & 0
\end{array}\right]
$$

sendo $x \in \mathbb{R}^{n}$ o vetor de estado, $u \in \mathbb{R}^{m}$ a entrada de controle, $w \in \mathbb{R}^{q}$ a entrada exógena, $z \in \mathbb{R}^{v}$ a saída controlada e $y \in \mathbb{R}^{p}$ a saída medida. Além disso, suponha que a matriz de transferência de $u$ para $y$ seja

$$
P_{22}=\left[\begin{array}{c|c}
A & B_{u} \\
\hline C_{y} & 0
\end{array}\right]
$$

com os pares $\left(A, B_{u}\right)$ e $\left(A, C_{y}\right)$ estabilizáveis e detectáveis, respectivamente. Então, a fatoração duplamente coprima é dada por

$$
P_{22}=N_{r} M_{r}^{-1}=\tilde{M}^{-1} \tilde{N}
$$

correspondendo a fatoração coprima à direita e à esquerda de $P_{22}$. Tal fatoração pode ser obtida a partir da identidade de Bezout generalizada

$$
\left[\begin{array}{cc}
\tilde{V}_{0} & -\tilde{U}_{0} \\
-\tilde{N} & \tilde{M}
\end{array}\right]\left[\begin{array}{ll}
M_{r} & U_{0} \\
N_{r} & V_{0}
\end{array}\right]=\left[\begin{array}{ll}
I & 0 \\
0 & I
\end{array}\right]
$$


Desse modo, a parametrização de um controlador estabilizante pode ser encontrada a partir dos seguintes fatores coprimos

$$
\begin{aligned}
K & =\left(U_{0}+M_{r} Q_{r}\right)\left(V_{0}+N_{r} Q_{r}\right)^{-1} \\
& =\left(\tilde{V}_{0}+Q_{l} \tilde{N}\right)^{-1}\left(\tilde{U}_{0}+Q_{l} \tilde{M}\right)
\end{aligned}
$$

com $Q_{r}$ e $Q_{l} \in R \mathcal{H}_{\infty}$ sendo o parâmetro de Youla (Zhou et al., 1996). Uma forma particular de determinar o controlador $K$ em espaço de estados pode ser definida como

$$
\left[\begin{array}{cc}
M_{r} & U_{0} \\
N_{r} & V_{0}
\end{array}\right]=\left[\begin{array}{c|cc}
A+B_{u} F & B_{u}-H \\
\hline F & I & 0 \\
C_{y} & 0 & I
\end{array}\right]
$$

$$
\left[\begin{array}{cc}
\tilde{V}_{0} & -\tilde{U}_{0} \\
-\tilde{N} & \tilde{M}
\end{array}\right]=\left[\begin{array}{c|cc}
A+H C_{y} & -B_{u} & H \\
\hline F & I & 0 \\
C_{y} & 0 & I
\end{array}\right]
$$

sendo que $A+B_{u} F$ e $A+H C_{y}$ devem ser estáveis. Aqui, o problema de controle usando a parametrização de Youla consiste basicamente em determinar uma matriz de ganho $K$ estabilizante a partir da lei de realimentação de saída $u=-K y$ tal que o sistema em malha fechada $T_{z w}$ garanta uma norma $\mathcal{H}_{\infty}$ menor ou igual que $\gamma$ (Fig. 3 ). Tal estratégia pode ser formulada e entendida a partir de algumas manipulações algébricas.

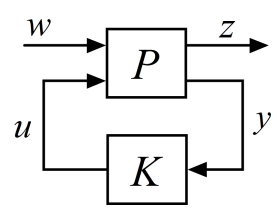

Figura 3. Diagrama de blocos do controle estabilizante com realimentação de saída.

A matriz de transferência em malha fechada $T_{z w}$ pode ser expressa na forma de uma transformação fracionária linear inferior (LFT) dada por

$$
T_{z w}=\mathcal{F}_{l}(P, K)=P_{11}+P_{12} K\left(I-P_{22} K\right)^{-1} P_{21}
$$

Fazendo $K\left(I-P_{22} K\right)^{-1}=-\left(U_{0}+M_{r} Q\right) \tilde{M}$, esta matriz pode ser escrita em termos do parâmetro $Q$,

$$
T_{z w}=\mathcal{F}_{l}(P, K)=P_{11}-P_{12} U_{0} \tilde{M} P_{21}-P_{12} M_{r} Q \tilde{M} P_{21}
$$

ou de forma equivalente

sendo

$$
T_{z w}=\mathcal{F}_{l}(T, Q)=T_{11}+T_{12} Q T_{21}
$$

$\left[\begin{array}{ll}T_{11} & T_{12} \\ T_{21} & T_{22}\end{array}\right]=\left[\begin{array}{cc|cc}A+B_{u} F & -B_{u} F & B_{w} & B_{u} \\ 0 & A+H C_{y} & B_{w}+H D_{w y} & 0 \\ \hline C_{z}+D_{u z} F & -D_{u z} F & D_{w z} & D_{u z} \\ 0 & C_{y} & D_{w y} & 0\end{array}\right]$

Consequentemente, o projeto de controle é dado em termos do parâmetro $Q$ que será utilizado no intuito de satisfazer alguns requisitos de desempenho. Assim, o problema de controle consiste em escolher um parâmetro $Q$ adequado tal que

$$
\left\|T_{z w}\right\|_{\infty}=\left\|\mathcal{F}_{l}(T, Q)\right\|_{\infty}=\left\|T_{11}+T_{12} Q T_{21}\right\|_{\infty} \leq \gamma
$$

seja satisfeito. Note que a matriz de transferência de malha fechada é afim do parâmetro $Q$ desde que $T_{22}=0$.

\section{PROJETO DE UM CONTROLADOR ESTABILIZANTE}

Nesta seção, apresenta-se um procedimento para o projeto de um controlador estabilizante usando a parametrização de Youla. A estratégia de controle proposta consiste na combinação da síntese dos ganhos $F$ e $H$ usando a norma $\mathcal{H}_{2}$ para a fatoração coprima e o uso da norma $\mathcal{H}_{\infty}$ para determinar o parâmetro $Q$.

\subsection{Controlador estabilizante}

Considere a estrutura geral dos controladores estabilizantes,

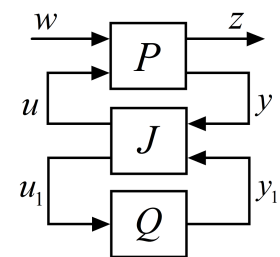

Figura 4. Representação de todos os controladores estabilizantes via parâmetro $Q$.

sendo

$$
\left[\begin{array}{c}
u \\
u_{1}
\end{array}\right]=J\left[\begin{array}{c}
y \\
y_{1}
\end{array}\right]
$$

Desta forma, o controlador estabilizante pode ser escrito como $K=\mathcal{F}_{l}(J, Q)$, ou seja

$$
u=\left[J_{11}+J_{12} Q\left(I-J_{22} Q\right)^{-1} J_{21}\right] y
$$

Sabendo que a parametrização de todos os controladores estabilizantes é dada por

$$
\begin{aligned}
K & =\left(U_{0}+M_{r} Q\right)\left(V_{0}+N_{r} Q\right)^{-1} \\
& =\left(\tilde{V}_{0}+Q \tilde{N}\right)^{-1}\left(\tilde{U}_{0}+Q \tilde{M}\right)
\end{aligned}
$$

tem-se que

$$
J=\left[\begin{array}{cc}
U_{0} V_{0}^{-1} & \tilde{V}_{0}^{-1} \\
V_{0}^{-1} & -V_{0}^{-1} N_{r}
\end{array}\right]
$$

que é equivalente a

$$
J=\left[\begin{array}{c|cc}
A+B_{u} F+H C_{y} & -H & B_{u} \\
\hline F & 0 & I \\
-C_{y} & I & 0
\end{array}\right]
$$

em espaço de estados. Agora, considerando que o parâmetro $Q$ seja definido como

$$
Q=\left[\begin{array}{c|c}
A_{Q} & B_{Q} \\
\hline C_{Q} & D_{Q}
\end{array}\right] \in R \mathcal{H}_{\infty}
$$

o controlador dinâmico estabilizante $K=\mathcal{F}_{l}(J, Q)$ pode ser escrito como sendo

$K=\left[\begin{array}{cc|c}A+B_{u} F+H C_{y}-B_{u} D_{Q} C_{2} & B_{u} C_{Q} & -H+B_{u} D_{Q} \\ -B_{Q} C_{y} & A_{Q} & B_{Q} \\ \hline F-D_{Q} C_{y} & C_{Q} & D_{Q}\end{array}\right]$

Diante do exposto, a determinação do controlador dinâmico estabilizante consiste em definir uma estratégia para obter o parâmetro $Q$ e as matrizes que compõem a fatoração duplamente coprima. Tal estratégia é apresentada a seguir. 


\subsection{Sintese da fatoração coprima e do parâmetro $Q$}

Aqui, tanto a síntese das matrizes $F$ e $H$ que compõem a fatoração duplamente coprima quanto a determinação do parâmetro $Q$ serão dadas a partir de condições em LMIs para a norma $\mathcal{H}_{2}$.

Teorema 1. Suponha que o par $\left(A, B_{u}\right)$ seja estabilizável. Então existe uma fatoração coprima à direita $P_{22}=$ $N_{r} M_{r}^{-1}$,

$$
\left[\begin{array}{c}
M_{r} \\
N_{r}
\end{array}\right]=\left[\begin{array}{c|c}
A+B_{u} F & B_{u} \\
\hline F & I \\
C_{y} & 0
\end{array}\right]
$$

com ganho $F=Z W^{-1} \in \mathbb{R}^{m \times n}$, se existem uma matriz simétrica $W \in \mathbb{R}^{n \times n}$ e as matrizes $Z \in \mathbb{R}^{m \times n}, X=X^{T} \in$ $\mathbb{R}^{n \times n}$ que satisfazem as seguintes desigualdades

$$
\begin{aligned}
& \operatorname{Tr}(X) \leq \mu_{r}^{2} \\
& \left(\begin{array}{cc}
X & I \\
\bullet & W
\end{array}\right)>0 \\
& \underset{<0}{\left(\begin{array}{ccc}
A W+W A^{T}+B_{u} Z+Z^{T} B_{u}^{T} & Z^{T} & W C_{y}^{T} \\
\bullet & -I & 0 \\
\bullet & \bullet & -I
\end{array}\right)}
\end{aligned}
$$

Prova. Considere um sistema em malha fechada definido por $T_{S F}=\mathcal{F}_{l}\left(P_{S F}, F\right)$, sendo

$$
P_{S F}=\left[\begin{array}{c|c|c}
A & I & B_{u} \\
\hline 0 & 0 & I \\
C_{y} & 0 & 0 \\
\hline I & 0 & 0
\end{array}\right]
$$

uma descrição particular para o problema de realimentação de estados (Zhou et al., 1996). O sistema em malha fechada $T_{S F}$ resulta em

$$
T_{S F}:=\left[\begin{array}{c|c|c}
\overbrace{A+B F}^{A_{M F}} & \overbrace{I}^{B_{M F}} \\
\hline \underbrace{F}_{C_{M F}} & \underbrace{\left[\begin{array}{c}
0 \\
0
\end{array}\right]}_{D_{M F}}
\end{array}\right] .
$$

Determinando a norma $\mathcal{H}_{2}$ do sistema em malha fechada $T_{S F},\left\|T_{S F}\right\|_{2} \leq \mu_{r}$, via Gramiano

$$
\begin{gathered}
\operatorname{Tr}(X) \leq \mu_{r}^{2} \\
\left(\begin{array}{cc}
X & B_{M F}^{T} \\
\bullet & W
\end{array}\right)>0 \\
\left(\begin{array}{ccc}
A_{M F} W+W A_{M F}^{T} & W C_{M F}^{T} \\
\bullet & & -I
\end{array}\right)<0
\end{gathered}
$$

e utilizando algumas manipulações algébricas, é possível obter o ganho $F$ que compõe a fatoração coprima à direita dada em (11), concluindo a prova.

Teorema 2. Suponha que o par $\left(A, C_{y}\right)$ seja detectável. Então existe uma fatoração coprima à esquerda $P_{22}=$ $\tilde{M}^{-1} \tilde{N}$,

$$
\left[\begin{array}{cc}
\tilde{M} & \tilde{N}
\end{array}\right]=\left[\begin{array}{c|cc}
A+H C_{y} & H & B_{u} \\
\hline C_{y} & I & 0
\end{array}\right]
$$

com ganho $H=V^{-1} Y \in \mathbb{R}^{n \times p}$, se existem uma matriz simétrica $V \in \mathbb{R}^{n \times n}$ e matrizes $Y \in \mathbb{R}^{n \times p}, \tilde{X}=\tilde{X}^{T} \in$ $\mathbb{R}^{n \times n}$ que satisfazem as seguintes desigualdades

$$
\begin{aligned}
& \operatorname{Tr}(\tilde{X}) \leq \mu_{o}^{2} \\
& \left(\begin{array}{cc}
\tilde{X} & I \\
\bullet & V
\end{array}\right)>0 \\
& \left.\begin{array}{cccc}
V A+A^{T} V+Y C_{y}+C_{y}^{T} Y^{T} & Y & V B_{u} \\
\bullet & \bullet & -I & 0 \\
<0 & \bullet & -I
\end{array}\right)
\end{aligned}
$$

Prova. Considere agora o sistema em malha fechada $T_{O I}=\mathcal{F}_{l}\left(P_{O I}, H\right)$, sendo

$$
P_{O I}=\left[\begin{array}{c|cc|c}
A & 0 & B_{u} & I \\
\hline I & 0 & 0 & 0 \\
\hline C_{y} & I & 0 & 0
\end{array}\right]
$$

uma descrição especial para o problema de injeção de saída (Zhou et al., 1996). Consequentemente, tem-se que

$$
T_{O I}=\left[\begin{array}{c|c}
\overbrace{A+H C_{y}}^{A_{M F}} & \overbrace{\left[\begin{array}{ll}
H B_{u}
\end{array}\right]}^{B_{M F}} \\
\hline \underbrace{I}_{C_{M F}} & \underbrace{[}_{D_{M F}\left[\begin{array}{ll}
0 & 0
\end{array}\right]}
\end{array}\right] .
$$

A prova é direta usando o mesmo procedimento empregado para a fatoração coprima à direita.

Agora, a estratégia consiste em escolher um parâmetro $Q$ adequado tal que

$$
\left\|T_{z w}\right\|_{\infty}=\left\|\mathcal{F}_{l}(T, Q)\right\|_{\infty}=\left\|T_{11}+T_{12} Q T_{21}\right\|_{\infty} \leq \gamma
$$

seja satisfeito. Desse modo, a planta generalizada $T$ pode ser derivada como

$$
T=\left[\begin{array}{c|cc}
A_{t} & B_{1} & B_{2} \\
\hline C_{1} & D_{11} & D_{12} \\
C_{2} & D_{21} & D_{22}
\end{array}\right]
$$

que é equivalente a

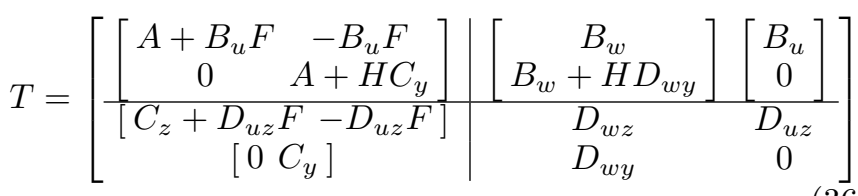

Usando condições em LMIs derivadas em (Scherer et al., 1997) para determinar um controlador $\mathcal{H}_{\infty}$ dinâmico por realimentação de saída, é possível fornecer condições necessárias e suficientes para a existência do parâmetro (sistema) $Q$ que satisfaz o critério de desempenho definido em (34).

Teorema 3. Considere a planta generalizada (35). Existe um parâmetro de Youla tal que $\left\|T_{z w}\right\|_{\infty} \leq \gamma$, se e somente se existir as matrizes $R=R^{T}>0, S=S^{T}>0, F_{t}, L, G$ e $D_{Q}$ que satisfazem as seguintes desigualdades

$\min \gamma^{2}$

$$
\begin{aligned}
& {\left[\begin{array}{ll}
S & I \\
I & R
\end{array}\right]>0} \\
& {\left[\begin{array}{c|c|c}
\Psi+\Psi^{T} & \left(\begin{array}{c}
B_{1}+B_{2} D_{Q} D_{21} \\
R B_{1}+L D_{21}
\end{array}\right) & \left(\begin{array}{c}
S C_{1}^{T}+F_{t}^{T} D_{12}^{T} \\
C_{1}^{T}+C_{2}^{T} D_{Q}^{T} D_{12}^{T}
\end{array}\right) \\
\hline \bullet & -\gamma^{2} & D_{11}^{T}+D_{21}^{T} D_{Q}^{T} D_{12}^{T} \\
\hline \bullet & \bullet & -I
\end{array}\right]}
\end{aligned}
$$


sendo

$$
\Psi=\left[\begin{array}{cc}
A_{t} S+B_{2} F_{t} & A_{t}+B_{2} D_{Q} C_{2} \\
G & R A_{t}+L C_{2}
\end{array}\right]
$$

Prova. A prova deste teorema é semelhante à desenvolvida em (Scherer et al., 1997).

Utilizando as variáveis $G, R, S, F_{t}, L$ e $D_{Q}$ encontradas via otimização, podemos determinar o parâmetro de Youla:

$$
Q:=\left[\begin{array}{c|c}
A_{Q} & B_{Q} \\
\hline C_{Q} & D_{Q}
\end{array}\right]
$$

sendo

$$
\begin{aligned}
& A_{Q}=\left(N^{T}\right)^{-1}\left(G-R A_{t} S-R B_{2} F_{t}-N^{T} B_{Q} C_{2} S\right) M^{-1} \\
& B_{Q}=\left(N^{T}\right)^{-1}\left(L-R B_{2} D_{Q}\right) \\
& C_{Q}=\left(F_{t}-D_{Q} C_{2} S\right) M^{-1}
\end{aligned}
$$

Adotando $N=I$ (não interfere no desempenho) (Scherer et al., 1997), pode-se encontrar $M$ a partir da equação

$$
M N^{T}=I-R S
$$

Com o parâmetro de Youla determinado, basta substituirmos as matrizes $A_{Q}, B_{Q}, C_{Q}$ e $D_{Q}$ na estrutura proposta para o controlador

$K=\left[\begin{array}{cc|c}A+B_{u} F+H C_{y}-B_{u} D_{Q} C_{2} & B_{u} C_{Q} & -H+B_{u} D_{Q} \\ -B_{Q} C_{y} & A_{Q} & B_{Q} \\ \hline F-D_{Q} C_{y} & C_{Q} & D_{Q}\end{array}\right]$

Por fim, um procedimento de projeto para obter o controlador estabilizante proposto pode ser apresentado:

(1) Resolva as LMIs (26) e (31) para encontrar os ganhos $F$ e $H$ que compõem a fatoração duplamente coprima, respectivamente.

(2) Monte a planta generalizada $T$ e resolva a LMI (37) para encontrar as matrizes $G, R, S, F_{t}, L$ e $D_{Q}$.

(3) Determine as matrizes $A_{Q}, B_{Q}, C_{Q}$ a partir das expressões (40).

(4) Monte o controlador estabilizante $K$ dado em (42).

\section{PROJETO E RESULTADOS EXPERIMENTAIS}

No intuito de avaliar a eficácia da proposta, as seguintes especificações foram definidas para o projeto de controle da planta: (1) garantir estabilidade para o problema de controle do nível; (2) erro de regime nulo para entrada ao degrau; e (3) rejeição de distúrbios com erro não superior a $10 \%$. Além disso, de forma a tornar a implementação mais simples, o modelo da planta industrial foi reduzido de $4^{\circ}$ para $2^{\circ}$ ordem e um pré-compensador $W_{s}$ foi utilizado

$$
W_{s}=\left[\begin{array}{cc}
\frac{s+0,2}{s} & 0 \\
0 & \frac{s+0,1}{s}
\end{array}\right]
$$

aumentando os ganhos em baixas frequências e o tipo do sistema. Agora, seguindo o procedimento de projeto apresentado, o primeiro passo consiste na determinação das fatorações coprimas à direita e à esquerda usando a norma $\mathcal{H}_{2}$. Resolvendo as LMIs em (26) o ganho $F$ encontrado que compõe a fatoração coprima à direta é

$$
F=\left[\begin{array}{cccc}
0,1475 & -0,1758 & -0,1389 & -0,0058 \\
0,1993 & 0,1309 & -0,0043 & -0,2114
\end{array}\right]
$$

com $\left\|T_{S F}\right\|_{2}=1,6925$, enquanto que $H$ em (31) que compõe a fatoração coprima à esquerda resultou em

$$
H^{T}=\left[\begin{array}{cccc}
0,2193 & -0,2752 & -0,5000 & -0,0012 \\
0,2745 & 0,1869 & 0,0024 & -0,2500
\end{array}\right]
$$

com $\left\|T_{O I}\right\|_{2}=2,8536$. Definindo a nossa planta generalizada $P$

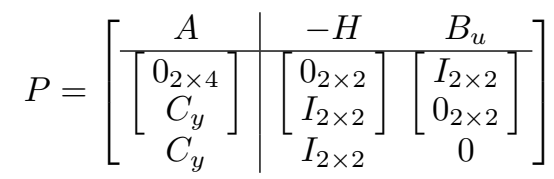

tem-se consequentemente (36). Resolvendo agora (37), o parâmetro $Q \in \mathbb{R}^{8 \times 8}$ é obtido com $\left\|T_{z w}\right\|_{\infty}=2,1290$. Depois de ter obtido o parâmetro $Q$, o controlador estabilizante $K$ em (24) pode ser construído, finalizando o procedimento apresentado. Entretanto, a ordem do controlador estabilizante ficou elevada para implementação prática. De forma a contornar esse problema, reduzimos a ordem do controlador estabilizante encontrado tal que o desempenho apresentado fosse bastante similar ao desempenho do controlador sem redução de ordem.

Para a implementação no sistema de nível, o controlador estabilizante de ordem reduzida foi discretizado usando um período de amostragem de $T_{s}=1[s]$. As seguintes equações a diferenças foram usadas para os sinais de entrada em termos do erro:

$u_{1}(k)=u_{1}(k-1)+2,999 e_{1}(k)-2,892 e_{1}(k-1)+\ldots$ $+0,1958 e_{2}(k)-0,1978 e_{2}(k-1)$

$u_{2}(k)=u_{2}(k-1)+0,1223 e_{1}(k)-0,1224 e_{1}(k-1)+\ldots$ $+1,863 e_{2}(k)-1,807 e_{2}(k-1)$

O experimento consistiu em rastrear as mudanças simultâneas do ponto de operação para os níveis de TQ1 e TQ2, apesar da perturbação - a partir do ponto de equilíbrio $13-18 \%$ para o TQ1 e a partir de $21-26 \%$ para o TQ2. Uma perturbação foi aplicada fechando parcialmente as válvulas, o que resulta em alterações no escoamento não modeladas. Os resultados experimentais são mostrados nas Figuras 5, 6, 7 e 8 .

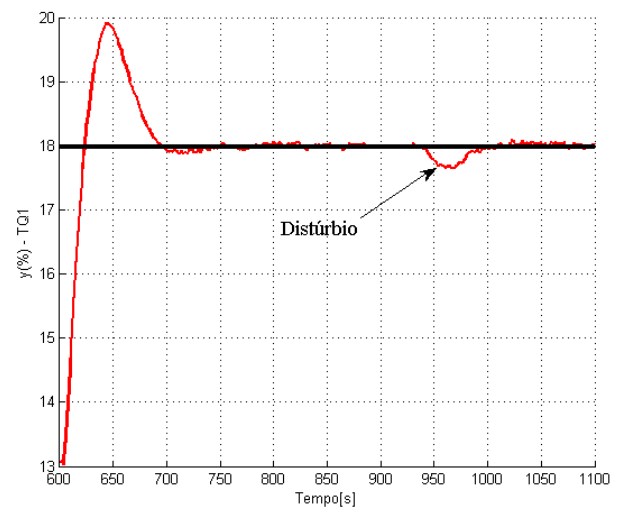

Figura 5. Resposta do sistema controlado para o TQ1.

Constata-se que o rastreamento da referência é alcançado apesar do distúrbio, sem erro de estado estacionário e com rejeição de distúrbios (erro) que não excede $10 \%$ para as saídas em ambos os pontos de operação, satisfazendo os requisitos impostos. 


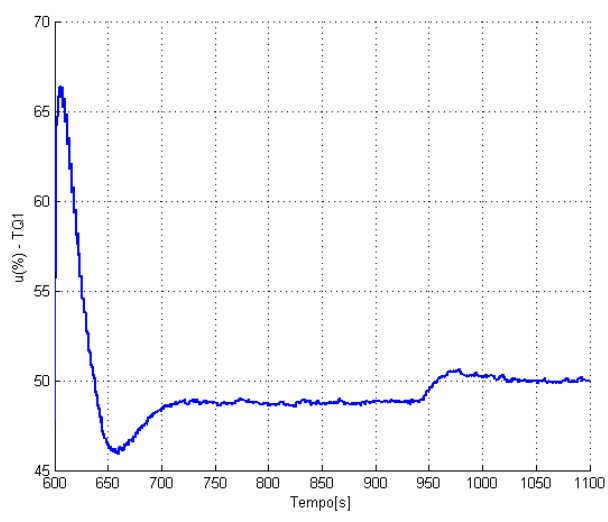

Figura 6. Esforço de controle aplicado para TQ1.

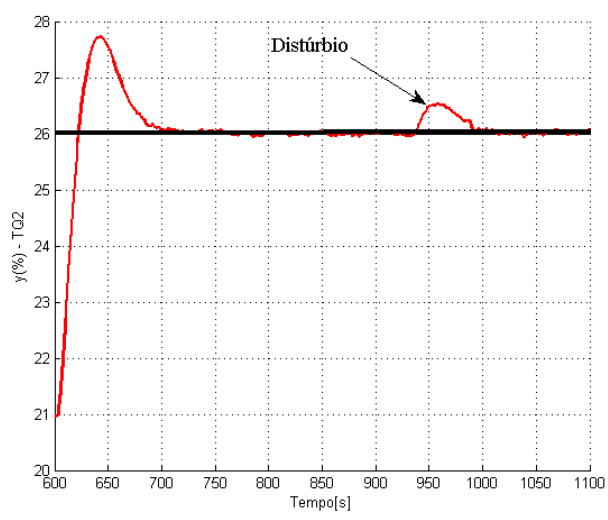

Figura 7. Resposta do sistema controlado para o TQ2.

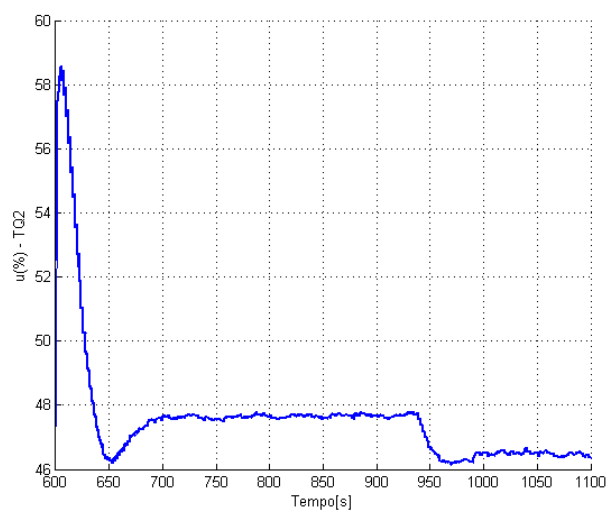

Figura 8. Esforço de controle aplicado para TQ2.

\section{CONCLUSÃO}

Neste artigo, apresentou-se uma implementação prática de um controlador estabilizante via parametrização de Youla em uma planta industrial de nível produzida pela empresa Yokogawa ${ }^{\circledR}$. Além disso, condições necessárias e suficientes em LMIs para síntese da fatoração duplamente coprima e do parâmetro $Q$ foram derivadas. Esta estratégia conduz a resultados satisfatórios que facilmente podem ser obtidos através de qualquer resolvedor. Ademais, adições de précompensadores e pós-compensadores podem ser necessárias no intuito de evitar sinais de controle inapropriados, indesejados efeitos de distúrbio e erros estacionários. Finalmente, os resultados experimentais obtidos mostraram que o controlador estabilizante pode ser uma alternativa interessante para o controle de nível em plantas industriais.

\section{REFERÊNCIAS}

Chen, G. and Han, Z. (1998). Robust right coprime factorization and robust stabilization of nonlinear feedback control systems. IEEE Transactions on Automatic Control, 43(10), 1505-1509.

Glover, K. and McFarlane, D. (1989). Robust stabilization of normalized coprime factor plant descriptions with $\mathcal{H}_{\infty}$-bounded uncertainty. IEEE Transactions on Automatic Control, 34(8), 821-830.

Kabamba, P.T., Meerkov, S.M., and Poh, E.. (1994). Youla parameterization in closed-loop vibrational control. IEEE Transactions on Automatic Control, 39(7), $1455-1459$.

Marx, B., Koenig, D., and Georges, D. (2003). Robust fault diagnosis for descriptor systems - a coprime factorization approach. IFAC Proceedings Volumes, 36(5), $477-482$.

McFarlane, D. and Glover, K. (1992). A loop-shaping design procedure using $\mathcal{H}_{\infty}$ synthesis. IEEE Transactions on Automatic Control, 37(6), 759-769.

McFarlane, D., Glover, K., and Vidyasagar, M. (1990). Reduced-order controller design using coprime factor model reduction. IEEE Transactions on Automatic Control, 35(3), 369-373.

Meyer, D.G. (1990). Fractional balanced reduction: model reduction via fractional representation. IEEE Transactions on Automatic Control, 35(12), 1341-1345.

Moore, J.B., Glover, K., and Telford, A. (1990). All stabilizing controllers as frequency-shaped state estimate feedback. IEEE Transactions on Automatic Control, 35(2), 203-208.

Moraes, A.T. and Kienitz, K.H. (2017). Robust control of an industrial pilot plant. IEEE Latin America Transactions, 15(9), 1613-1620.

Niemann, H. (2003). Dual Youla parameterisation. IEE Proceedings - Control Theory and Applications, 150(5), 493-497.

Pereira, R.L., Kienitz, K.H., and d. Moraes, A.T. (2016). $\mathcal{H}_{\infty}$ loop shaping control under parametric and nonparametric uncertainties: A case study. Journal of Control, Automation and Electrical Systems, 27(5), 506-514.

Prempain, E. and Postlethwaite, I. (2005). Static $\mathcal{H}_{\infty}$ loop shaping control of a fly-by-wire helicopter. Automatica, 41(9), $1517-1528$.

Scherer, C., Gahinet, P., and Chilali, M. (1997). Multiobjective output-feedback control via LMI optimization. IEEE Transactions on Automatic Control, 42(7), 896911.

Verma, M.S. and Hunt, L.R. (1993). Right coprime factorizations and stabilization for nonlinear systems. IEEE Transactions on Automatic Control, 38(2), 222231.

Youla, D., Jabr, H., and Bongiorno, J. (1976). Modern wiener-hopf design of optimal controllers-part II: The multivariable case. IEEE Transactions on Automatic Control, 21(3), 319-338.

Zhou, K., Doyle, J.C., and Glover, K. (1996). Robust and Optimal Control. Prentice-Hall, Inc., Upper Saddle River, NJ, USA. 